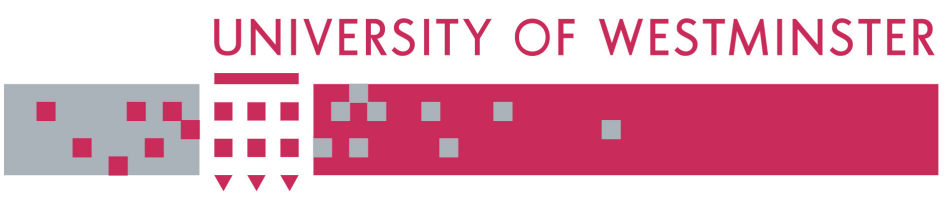

WestminsterResearch

http://www.wmin.ac.uk/westminsterresearch

\title{
A novel generic distributed intelligent re-configurable mobile network architecture.
}

\author{
R.E. Ramos \\ Kambiz Madani
}

Cavendish School of Computer Science

Copyright (C) [2001] IEEE. Reprinted from 2001 IEEE 52nd Vehicular Technology Conference: VTC 2001 - Spring, pp. 1927-1931.

This material is posted here with permission of the IEEE. Such permission of the IEEE does not in any way imply IEEE endorsement of any of the University of Westminster's products or services. Internal or personal use of this material is permitted. However, permission to reprint/republish this material for advertising or promotional purposes or for creating new collective works for resale or redistribution must be obtained from the IEEE by writing to pubs-permissions@ieee.org. By choosing to view this document, you agree to all provisions of the copyright laws protecting it.

The WestminsterResearch online digital archive at the University of Westminster aims to make the research output of the University available to a wider audience. Copyright and Moral Rights remain with the authors and/or copyright owners. Users are permitted to download and/or print one copy for non-commercial private study or research. Further distribution and any use of material from within this archive for profit-making enterprises or for commercial gain is strictly forbidden.

Whilst further distribution of specific materials from within this archive is forbidden, you may freely distribute the URL of WestminsterResearch.

(http://www.wmin.ac.uk/westminsterresearch).

In case of abuse or copyright appearing without permission e-mail wattsn@wmin.ac.uk. 


\title{
A Novel Generic Distributed Intelligent Re-configurable Mobile Network Architecture
}

\author{
R. E. Ramos, K. Madani. \\ Westminster University, 115 New Cavendish Street, London W1W 6UW, England. \\ r.ramos@cmsa.westminster.ac.uk, madani@cmsa.westminster.ac.uk
}

\begin{abstract}
In this paper we present our vision of the Software Radio (SR) concept. To the author's knowledge this vision of an Intelligent Re-configurable Network encompasses and adds to the main SR concepts presented to date, namely SR/SDR, Re-configurable radio systems and networks, and Cognitive radio. The newly proposed architecture and supporting technology provides a flexible way to introduce re-configurability into any network node and protocol layer of a network fixed or mobile, while simultaneously providing a structured framework in which re-configurability can be introduced and hence networks can evolve. This concept has provided a solid foundation and plays a central role in providing a suitable context in which to examine reconfigurabilty issues in the Mobile Terminal and Base Station. We believe that this concept has the potential to help the telecommunications industry in facilitating; the introduction, development and harmonization of reconfigurability within existing and future fixed and mobile networks.
\end{abstract}

\section{Introduction}

Our vision of the Software Radio (SR) is of an intelligent re-configurable mobile terminal and serving mobile network. It extends on the idea of Mitola's SR concept [1-5], in that is considers low implementation complexity re-configurability the salient issue and not just defining radio functionality in software. It also differs from this definition by embracing the idea of re-configurability not only in the mobile terminal but also in the serving mobile network, as in the work on Re-configurable radio systems and networks [6-8]. Our work then further extends on this idea by considering the necessary technology to support intelligent reconfiguration, through the use of model-based automated reasoning and a Network Reconfiguration Language (NRL), addressing similar problems as the cognitive radio concept [9-10]. However, our work is not just restricted to the terminal but applied to the serving mobile network as well. In this paper we start with a generic definition of intelligent reconfigurable networks, and apply the essential principles of this to $2^{\text {nd }}$ and $3^{\text {rd }}$ generation mobile radio networks. This allows us to build a definition of a generalized reconfigurable mobile architecture and identify the essential technology it requires to support reconfiguration. This definition is playing a central role in our research investigations into re-configurability in existing and future networks carried out in the EC Framework V CAST project [11].

We believe, that the essential principles that have been used to build the newly proposed architecture provide a way to introduce re-configurability into any network node and protocol layer of a fixed or mobile network. This provides flexibility but at the same time a structured framework in which re-configurable networks can evolve. Hence it could potentially be useful to the telecommunication industry in facilitating; the introduction, development and harmonization of reconfigerability within existing and future fixed and mobile networks. In this paper we focus on its application to mobile networks, as we are concentrating on case studies based on GSM 1800 and UMTS UTRA.

\section{Intelligent Re-configurable Network}

The essential elements of a re-configurable network are illustrated in figure 1.0. This diagram shows that we have a re-configurable network that consists of reconfigurable hardware and software modules. To reconfigure any part of the network it is necessary for the network to have some intelligence and re-configuration control. The intelligence is used to decide what part or parts of the network should be re-configured, based on relevant information supplied to it, it then gets the reconfiguration controller to implement these decisions on the appropriate hardware and/or software modules. It is important to note that in our definition that the concept of re-configurability is applicable to all varieties of networks, fixed or mobile. In this paper we are considering mobile networks, and more specifically using 
case studies of GSM 1800 and UMTS UTRAN as the foundation for intelligent re-configurability. The most essential information for this generic definition is presented here, and further details can be found in reference [12]

\section{Mobile Network Architecture}

We define the Mobile Network to consist of two basic elements the:

- Mobile User Terminal and

- Serving Mobile network.

What we mean by Mobile Radio User Terminal is very clear, but the Serving Mobile Network needs further clarification. The Serving Mobile Network is defined as the network elements that facilitate mobile radio communication.

The scope of the network architecture examined and the level of detail to which it would be necessary to investigate is illustrated in figure 2.0 , labeled Reconfigurable Mobile Network Architecture. The mobile network architecture is of course common to both GSM 1800 and UMTS UTRA even though the air-interfaces are completely different.

Although we are examining re-configurability issues and enabling technologies in the handset and the base station, it is necessary to study the hardware and protocol functionality all the way up-to the mobile network elements. Applying the definitions of Serving mobile network, here the serving network elements consist of Base Transceiver Stations (BTS), Base Station Controllers (BSC), and Mobile Switching Center (MSC), as these are the network elements that facilitate mobile communication.

As our approach, in this research, is to examine intelligent re-configurability issues in the whole Mobile Network in relation to Terminal and Base Station, it is necessary to examine the Software (protocols) and hardware functional building blocks for all Network nodes and Protocol layers. Here the network nodes are the MS, BTS, BSC and MSC, as illustrated in Figure 2.0.

The purpose is to from a functional point of view to identify generic hardware and software/protocol platforms and potential areas for re-configurable software/protocol and hardware.

\section{Intelligent Re-configurable Mobile Network Architecture (IRMNA)}

We will now present our vision of an Intelligent Reconfigurable Mobile Network Architecture. Figure 2.0 displays the essential components of an IRMNA. These are:

- Global and Local Intelligent Re-configurable Controllers

- A Re-configurable Mobile Network

- A Network Re-configuration Language (NR language)

- A software down load mechanism

To Re-configure a network intelligently, it is necessary for the terminal and network to be aware of the:

- End user Environment (application profiles etc)

- Radio Environment,

- Network Environment (i.e. the current state of the different hardware and software components of the nodes and protocol layers of the network).

It is obvious that it will be necessary for the network to have quiet a sophisticated information gathering engine, and processing capability, in order to make intelligent decisions about re-configuration. Our definition of the SR concept reflects this fundamental understanding.

We will now explain the function of the basic network elements to gain an overall understanding of how the systems works.

\subsection{Global Intelligent Re-configurable Controller (GIRC)}

The GIRC consists of a Network Manager, Intelligence, a Database, and a Re-configuation Controller. GIRC along with the Local Intelligent Reconfigurable Controllers (to be discussed later), are considered the heart of the Intelligent Re-configerable Technology. It gathers the following information:

- from the reconfigurable mobile network, regarding the state of different hardware and software components in the different nodes and protocol layers;

- the end user applications, regarding the user profiles and environment;

- the radio environment, via the Network Manager. 
This is stored in the database, were it may be further processed and filtered. The intelligence, working in conjunction with the database, then makes a decision or decisions on what needs to be re-configured. A modelbased automated approach will be used to implement the intelligence. We are considering the CODA technology [13] to implement the intelligence, but in principle any intelligence technology that is appropriate can be deployed.

The re-configuration decisions are then passed to the network manager who then passes this information to the Re-configurable Controller who's function it is to ensure that the re-configuration decision is implement, and if there is any problems to inform the Network Manager. The Re-Configurable Controller then passes the reconfiguration decision to the Local Intelligent Reconfiguration Controller, in the appropriate network node and protocol layer, were a the request for reconfiguration will be implemented on the relevant reconfigurable hardware and/or software. If it does not agree with the request it will inform the Global Reconfiguration Controller. There are two types of reconfiguration Globally Instigated and Locally Instigated. This is an example of a globally instigated reconfiguration. In a locally instigated Re-configuration the local Intelligent Reconfiguration Controller will identify the need for re-configuration in its particular node and protocol layer, and may execute this reconfiguration immediately if it is appropriate to make the decision locally (hence the distributed nature of network management and intelligence), or inform the Global Intelligence Re-configurable Controller for permission.

In general all information gathered form the Reconfigurable mobile network is passed to the Network manager who then passes it to the Database or Intelligence as necessary. Also all global instigated requests for re-configuration are passed from the Intelligence to the Network Manager who then passes it to the Re-configuration Controller, and like wise locally instigated requests for $\mathrm{Re}$-configuration are passed from the Re-configurable Controller to the Network Manager who then passes it to the Intelligence. So we can see that all relevant information to re-configuration from the mobile network and all requests for re-configuration go through the Network Manager, to ensure co-ordination and synchronization of re-configuration activities. The only time that the Network Manager is by-passed is if there is an emergency re-configeration, in this case the Intelligence directly informs the Re-configuration Controller to save time.
As we can see, the intelligence will be distributed between global and local, understanding how this will be apportioned is currently part of our research effort. Also, we are investigating how the Global Intelligent Reconfigurable Controller Technology will be incorporated into existing $2 \mathrm{G}, 3 \mathrm{G}$, and future mobile networks.

Note that an important distinction between Intelligent Re-configurable Technology and Re-configurable Enabling Technology is being made. As we feel that a lot of research effort is being made into Re-configurable Enabling Technology but very little into Intelligent Reconfigurable Technology. It is important to appreciate that without the latter the former will not work. Hence one of the main goals of this project is to contribute to the development of the Intelligent Re-configurable Technology with respect to re-configurability issues in the handset and basestation. This project is also developing a re-configurable Terminal and basesation demonstrator, which contributes to Re-configurable Enabling Technology, but the primary purpose of the demonstrator, is to test out the Intelligent $\mathrm{Re}$ configurable Technology concepts.

The Re-configurable Mobile Network, therefore, consists of the re-configurable network nodes; Mobile Terminal, Base Station, Base Station Controller and Mobile Switching Center, and the different protocol layers associated with these nodes. The interconnection of these nodes resembles the architecture of GSM1800 and UMTS UTRA because our research is based on case studies of these networks, even though they have completely different air-interfaces UMTS UTRA has been developed in such a way as to take advantage of existing GSM supporting network infrastructure.

\subsection{General structure of an Intelligent Re- configerable Layer (IRL) within a Node}

This is illustrated in figure 2.0 , and this structure is applicable to any layer. This figure shows that an IRL consists of a Local Intelligent Re-configuration Controller (LIRC), Fixed Hardware and Software platform (or platforms), and Re-configurable Hardware and Software Platform (or platforms). The LIRC has a similar internal architecture a functioning as the global one but is scaled down, as it only has to manage reconfiguration within its protocol layer and node. As explained in the previous section it works in co-operation with the GIRC to facilitate re-configuration within its protocol layer and node and can also make local independent intelligent decisions in appropriate 
circumstances. The LIRC basically co-ordinates the reconfiguration of the hardware and software components within the protocol layer and node. This is achieved by communicating with different re-configurable components to find out about their current state, using a Network Re-configuration Language (NRL). It is necessary to develop a NRL so that the re-configurable modules within a node and protocol layer can report necessary information about their state to the LIRC when required, the LIRC can make requests to re-configure, the LIRC can instruct software and hardware modules to re-configure, and the LIRC can communicate with the GIRC when necessary Object Oriented programming techniques will be used to ensure that user-friendly message interfaces can be built, and manufacturing implementation detail can be hidden. Allowing different manufactures to build the same re-configurable hardware or software modules. Re-configuration of these modules will be achieved by software downloading.

Software down loading is used to re-configure software and hardware module personalities within the mobile network, patch-up bugs, and up-grade software when new release come out.

\subsection{Conclusions}

The main aim of the this paper was to clearly define our vision and understanding of the Software Radio concept, so as to have a suitable context in which to examine reconfigerability issues in the Mobile Terminal and Base Station and their associated intelligent re-configurable technologies and enabling technologies.

In doing so we have made a decisive move away for emphasizing software radio and prefer to focus on intelligent re-configurability. We have embraced the idea of re-configurability in the whole mobile network, as opposed to just in the terminal, but added to this by identifying and defining the essential elements that facilitate intelligent decision making to support reconfiguration across all the network nodes and protocol layers for the networks under examination.

The proposed architecture is a flexible re-configurable mobile network that employs distributed (global and local) intelligence / network management and the essential technology to facilitate its re-configuration.

This is based on the generic definition of an intelligent re-configurable network presented in section 2.0. Note that this vision of an intelligent re-configurable network encompasses the main Software Radio concepts presented in section 1.0 . We believe that the fundamental definition can also be applied not only to any mobile network but also to any fixed network.

The newly proposed architecture and supporting technology provides a flexible way to introduce reconfigurability into any network node and protocol layer of a network. At the same time it provides a structured framework in which re-configurable networks can evolve. Therefore we believe it has the potential to help in facilitating; the introduction, development and harmonization of reconfigurability within the existing and the future fixed and mobile networks. We are currently refining this initial concept within the CAST project, the results of which will be presented in due course.

\section{Acknowledgement}

This document is based on work carried out in the EUsponsored collaborative research project CAST (http://www.cast5.freeserve.co.uk/). Nevertheless, only the authors are responsible for the views expressed here.

\section{References}

[1] Mitola J., "The Software Radio Architecture", IEEE Communications Magazine, pp26-38, May 1995.

[2] Mitola J., "Software Radio Architecture Evolution: Foundations, Technology Tradeoffs, and Architecture Implications", IEICE Trans. Commn., Vol. E83-B, No.6, pp 1165-1172, June 2000.

[3] Cummings M., Heath S., "Mode Switching and Software Download for Software Defined Radio: The SDR Forunm Approach", IEEE Communications Magazine, pl04-106, August 1999.

[4] Tuttlebee W., "Software Radio Technology: A European Perspective", IEEE Communications Magazine, p118-123, February 1999

[5] "WP 1.2 Architectural Functions", Section 8.1.2: The SR and SRD Concept, EC Framework VCAST project Public Report, Date of Delivery 30.9.2000, pp 43-44.

[6] Pereira J. M, "Re-Defining Software (Defined) Radio: ReConfigurable Radio Systems and Networks", IEICE Trans.

Commn., Vol. E83-B, No.6, , pp 1175-1182, June 2000.

[7] Pereira J. M et al, "Re-configurable Radio Systems \& Networks", Working Document Draft 2.0, First European Colloquium, London, $4^{\text {th }}$ March 19999.

[8] "WP 1.2 Architectural Functions", Section 8.1.8: ReConfigurable Radio Systems and Networks, EC Framework VCAST project Public Report, Date of Delivery 30.9.2000, pp 46-47.

[9] Mitola J., Maguire G., "Cognitive Radio: Making Software Radios More Personal", IEEE Personal Communications, pp 13-18. August 1999. 
[10] "WP 1.2 Architectural Functions", Section 8.1.8: Cognitive Radios, EC Framework V CAST project Report, Oct 2000, pp 47.

[11] K. Madani, B. Bosch, B. Honary, G. Justo, J. Kova'cs, M Lohi, D. Lund, P. Patel, R. Ramos, S. Imre, "Configurable radio with Advanced Software Technology (CAST) - Initial Concepts", IST Mobile Communications Summit 2000 Gallway Ireland, 1-4 Oct. 2000, pp 139-144.
[12] "WP 1.2 Architectural Functions", Section 8.1.10: Generic Definition of an Intelligent Re-configurable Network, EC Framework VCAST project Public Report, Date of Delivery 30.9.2000, pp48-49

[13] T. Karran, J. Gusto, K. Madani, "Intelligent Reconfiguration of Large Mobile Networks using a Complex Organic Distributed Architecture", accepted for presentation at the VTC Spring, May 6-9 2001

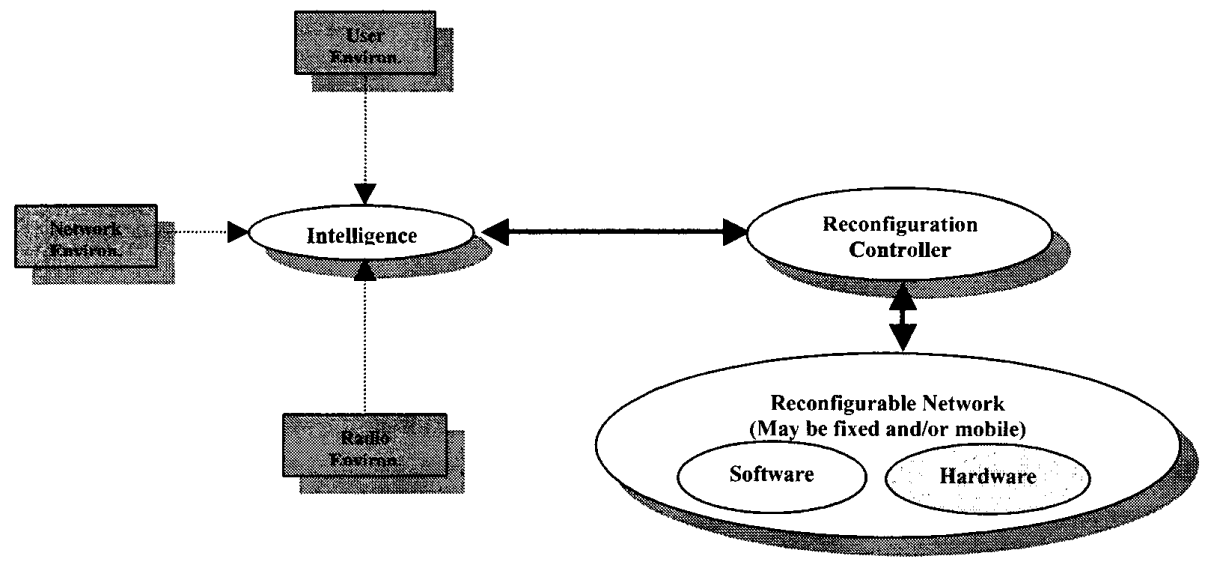

Figure 1.0: Essential elements of re-configurable network.

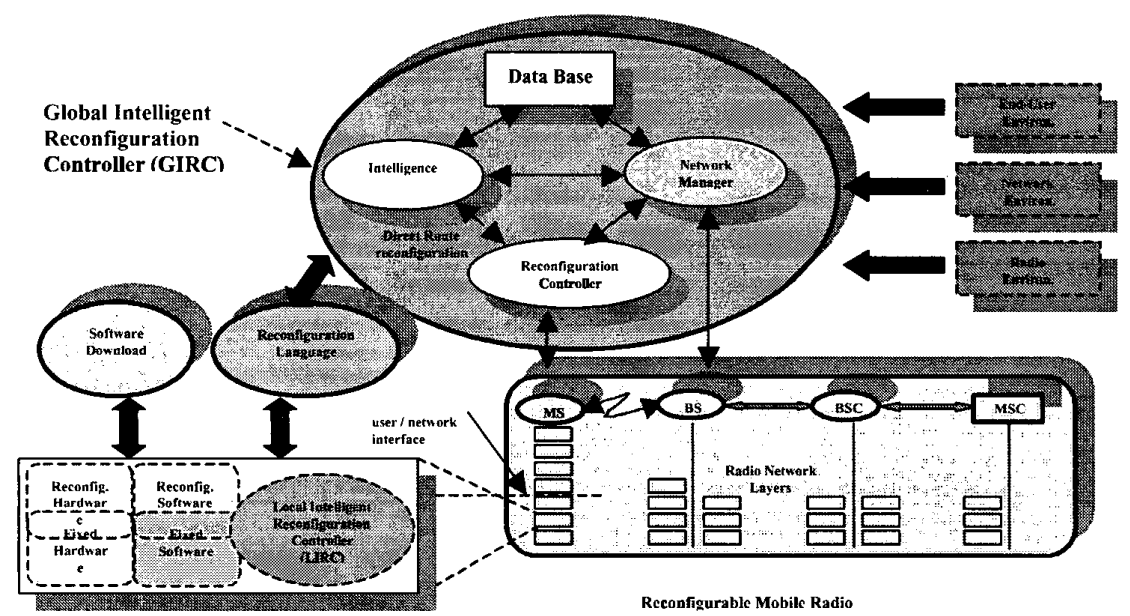

Intelliarnt Reconfiamrable I aver

Figure 2.0: Essential components of an intelligent reconfigurable mobile network architecture 